\title{
Ärzte vor dem Richter - Wann handeln Ärzte fahrlässig?
}

\author{
Was bedeutet Fahrlässigkeit im juristischen Sinne? Wann liegt eine Sorgfaltspflicht- \\ verletzung vor? Das Wissen darum ist unerlässlich, um den Mühlen der Strafjustiz \\ zu entgehen.
}

Philipp Juchli, Patrick Stach

Rechtsanwälte

Interaktiver
Artikel
Wollen Sie diesen Artikel
kommentieren? Nutzen
Sie dafür die Kommentar-
funktion in der Online-
Version oder sehen Sie
nach, was Ihre Kolleginnen
und Kollegen bereits
geschrieben haben:
www.saez.ch/
aktuelle-ausgabe/
interaktive-beitraege/

Korrespondenz:

Dr. iur. Philipp Juchli Stach Rechtsanwälte und Notare Poststrasse 17

CH-9001 St. Gallen

Tel. 0712787828

Fax 0712787829

juchli[at]stach.ch
Kaum eine Woche vergeht ohne Schlagzeilen, dass sich ein Arzt vor dem Richter zu verantworten hat. Den beschuldigten Ärzten wird regelmässig vorgeworfen, aufgrund von Eingriffsfehlern fahrlässig den Tod oder die Verletzung ihres Patienten herbeigeführt zu haben. Die Medien sorgen dafür, dass solche Verfahren in der breiten Öffentlichkeit bekannt werden. Die Funktion der Medien als «public watchdog» wird dabei zeitweise überinterpretiert, und die an den Pranger gestellten Ärzte werden auf persönlicher Ebene angegriffen und zur Schau gestellt.

In der Tat ist ärztliches Handeln durchwegs von rechtlichen Rahmenbedingungen geprägt. Da bei ärztlichen Eingriffen per definitionem die Abwehr von Beeinträchtigungen in Leib und Leben zu beurteilen ist, verwundert es aus juristischer Perspektive zunächst einmal nicht, dass nicht nur zivilrechtliche Aspekte zu beachten sind, sondern bei ärztlichen Fehlleistungen auch strafrechtliche Sanktionen, namentlich wegen Körperverletzung oder Tötung, drohen. Der vorliegende Beitrag beschränkt sich auf die Darstellung der strafrechtlichen Gefahren ärztlichen Handelns und soll als Leitfaden dienen, der Ärzteschaft die Tücken eines Strafverfahrens aufzuzeigen. Analoges gilt auch für Zivilprozesse.

\section{Der juristische Fahrlässigkeitsbegriff}

In Strafverfahren gegen Ärzte ist erfahrungsgemäss der Fahrlässigkeitsbegriff für die Frage zentral, ob ein Handeln strafrechtlich zu ahnden ist. In einem ersten Schritt ist deshalb zu erläutern, was mit Fahrlässigkeit im juristischen Sinne gemeint ist.

\section{Der Fahrlässigkeitsbegriff im Gesetz}

Gemäss Strafgesetzbuch handelt fahrlässig, wer die Folge seines Verhaltens aus pflichtwidriger Unvorsichtigkeit nicht bedenkt oder darauf nicht Rücksicht nimmt. Pflichtwidrig handelt, wer die Vorsicht nicht beachtet, zu der er nach den Umständen und nach seinen persönlichen Verhältnissen verpflichtet ist [1]. Selbst leichte Fahrlässigkeit löst die strafrechtliche Belangbarkeit aus. Da das Gericht die Strafe nach dem Verschulden des Täters zuzumessen hat

\section{Les médecins devant le juge: quand peut-on parler de négligence?}

Pas une semaine ne s'écoule sans que les médias ne parlent d'un médecin qui se retrouve devant le juge pour répondre de ses actes. On reproche en effet régulièrement aux médecins inculpés d'avoir causé un dommage à la santé ou provoqué la mort d'un patient par négligence à la suite d'une intervention. Les médias s'en font l'écho auprès d'un large public et surestiment parfois leur fonction de «gardiens de l'ordre public» en attaquant les médecins concernés sur le plan personnel et en faisant d'eux des exemples. En réalité, l'activité médicale est complètement encadrée par la loi. Par définition, une intervention médicale implique une atteinte au corps et à la vie du patient. Sur le plan juridique, il n'est donc guère surprenant au premier abord qu'il faille non seulement tenir compte des aspects de droit civil, mais également que des sanctions pénales pour lésions corporelles ou homicide menacent les médecins en cas de faute médicale. Le présent article se limite à la présentation des risques inhérents à l'activité médicale sur le plan pénal et a pour vocation de servir de guide aux médecins en les rendant attentifs aux pièges des procédures pénales. Les mêmes explications valent également pour les procédures civiles.

[2], kann leichte Fahrlässigkeit immerhin im Rahmen der Strafzumessung zugunsten des beschuldigten Arztes berücksichtigt werden. Indessen sind Ärzte nicht davor gefeit, vor den Richter gezogen zu 
werden, auch wenn ihnen lediglich eine geringfügige Verfehlung zur Last gelegt wird.

\begin{abstract}
Adäquanz
Hingegen ist - die Verantwortlichkeit einschränkend unsorgfältiges Verhalten gemäss ständiger Rechtspraxis lediglich dann anzunehmen, wenn die schadensbegründende Ursache geeignet ist, nach dem gewöhnlichen Lauf der Dinge und der Lebenserfahrung einen Erfolg wie den eingetretenen herbeizuführen oder mindestens zu begünstigen (sog. Adäquanztheorie) [3]. Vergisst beispielweise der operierende Arzt die Schere im Bauch des Patienten, und kommt es infolgedessen zu Komplikationen, so muss gefragt werden, ob nach dem gewöhnlichen Lauf der Dinge die vergessene Schere (Ursache) geeignet ist, den eingetretenen Erfolg, mithin die körperlichen Beschwerden, herbeizuführen. Wäre es nicht möglich oder dermassen unwahrscheinlich, dass die Schere die aufgetretenen Beschwerden herbeiführt, würde es an der Adäquanz fehlen und folglich müsste die strafrechtliche Haftung des Arztes entfallen.
\end{abstract}

len Kenntnissen und Fähigkeiten eines Arztes [4], wobei von diesem erwartet wird, dass die Anamnese und die Behandlung nach anerkannten Regeln der medizinischen Kunst erfolgt. Ein Arzt handelt folglich fahrlässig, wenn ihm entweder ein eigentlicher Kunstfehler unterläuft - sei es, dass ein notwendiger Eingriff nicht oder eine Behandlung nicht zeitgerecht erfolgt - oder bereits dann, wenn im Zuge der Diagnose Fehleinschätzungen passieren, die bei sorgfältiger Arbeitsweise nicht hätten passieren dürfen.

Bei Ärzten fällt besonders ins Gewicht, dass aufgrund ihrer Ausbildung sowie der beruflichen Erfahrung davon ausgegangen wird, dass sie über Fähigkeiten und Fachkenntnisse verfügen, um mögliche Gefahren für Patienten zu erkennen oder auszuschliessen. Bei Ärzten wird der Rahmen der zu berücksichtigenden «persönlichen» Verhältnisse daher auf das abstrahiert, was von ihrer Berufsgruppe erwartet werden kann [5], wobei allerdings etwaigen Spezialisierungen und besonderen Fachkenntnissen Rechnung zu tragen ist. Von einem Facharzt wird

\section{Wegen Fahrlässigkeit kann nur verantwortlich gemacht werden, wer «nach seinen persönlichen Verhältnissen» mit grösserer Sorgfalt hätte vorgehen können.}

\section{Vermeidbarkeit und Voraussehbarkeit}

$\mathrm{Zu}$ den Grundvoraussetzungen gehören immerhin die Voraussehbarkeit des Erfolgs (d. h. der Körperverletzung oder des Todes) und dessen Vermeidbarkeit, in der Regel durch das Ergreifen von Vorkehrungen, die das Risiko seiner Verwirklichung ausschliessen bzw. auf das erlaubte Mass reduzieren. Die an den Arzt gestellten Anforderungen an den Sorgfaltsmassstab dürfen selbstverständlich nicht weiter reichen als die menschliche Fähigkeit, Geschehensabläufe etwa den Krankheitsverlauf bei Komplikationen vorherzusehen und gestaltend auf sie Einfluss zu nehmen. Ist die durch den Arzt begangene Körperverletzung oder der von ihm verursachte Tod nicht voraussehbar, weil medizinisch schlichtweg nicht erkenn- oder vermeidbar gewesen, handelt der Arzt nicht fahrlässig, weshalb seine Strafbarkeit ausscheiden muss.

\section{Fahrlässigkeit und ärztliches Handeln}

\section{Persönliche Verhältnisse}

Wegen fahrlässiger Begehung einer Tat kann nur verantwortlich gemacht werden, wenn der Arzt «nach seinen persönlichen Verhältnissen» imstande gewesen wäre, mit grösserer Sorgfalt vorzugehen, als er es tatsächlich getan hat. Die strafrechtliche Verantwortlichkeit bemisst sich demnach nach individuel- demnach erwartet, dass er gegenüber einem Allgemeinpraktiker mehr fachspezifisches Wissen mitbringt und dieses auch anwenden kann. Im Sinne eines «Erst-recht-Argumentes» wenden deshalb die Gerichte bei Fachärzten tendenziell einen strengeren Sorgfaltsmassstab an.

Insbesondere eine unterbliebene Differentialdiagnose und damit verbunden die möglicherweise fatalen Folgen der unterlassenen Abklärung von Alternativursachen für Symptome können dem behandelnden Arzt zum Vorwurf gemacht werden. So wurde etwa ein Arzt der fahrlässigen Tötung schuldig gesprochen, weil bei einer Schwangerschaftskomplikation zunächst eine Stressgastritis und danach eine Eklampsie diagnostiziert wurde. Tatsächlich verstarb die Patientin am HELLP-Syndrom. Das Gericht erkannte auf diverse Sorgfaltspflichtverletzungen. So habe der Arzt die klinischen Leitsymptome einer hypertensiven Schwangerschaftserkrankung verkannt und das HELLP-Syndrom zu Unrecht nicht in die Differentialdiagnose einbezogen. Ausserdem hätte er die Anamnese und die Krankengeschichte berücksichtigen und die Patientin besser überwachen müssen [6]. Ein Schuldspruch erfolgte auch gegenüber einem Gynäkologen, der nach einer Totgeburt aufgrund von anhaltenden Blutungen an der Diagnose der Atonie festhielt, obwohl die Behandlung mittels Gebärmuttermassage nicht die ge- 


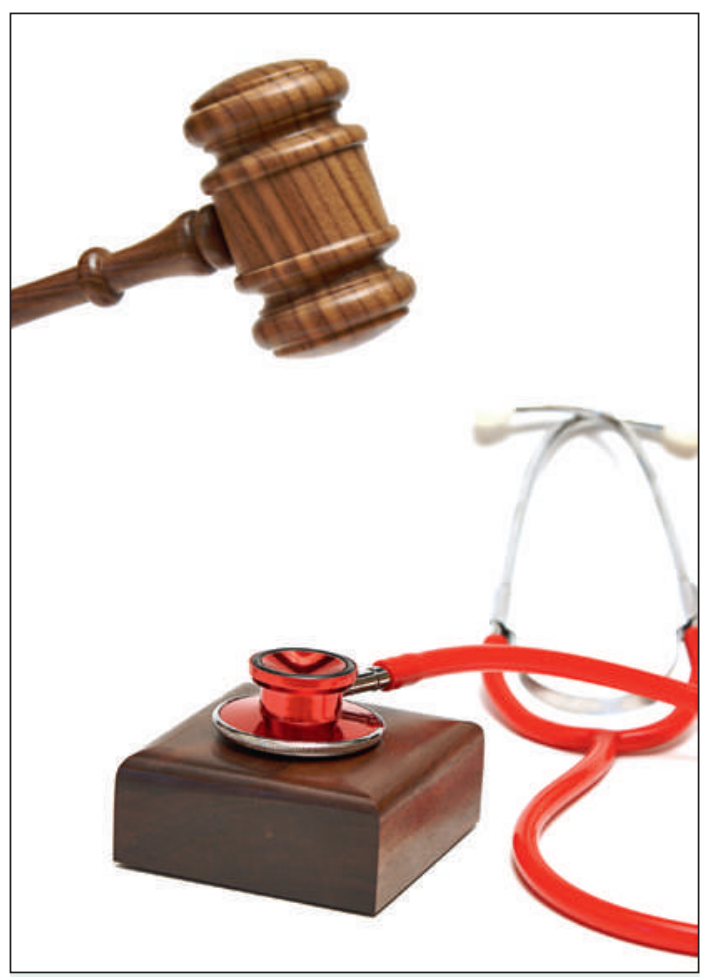

Der Hauptvorwurf bei Verfahren gegen Ärzte: wegen Eingriffsfehlern fahrlässig den Tod oder die Verletzung ihres Patienten herbeigeführt zu haben.

wünschte Wirkung zeigte. Die Patientin verstarb schliesslich an den Folgen einer nicht diagnostizierten Gebärmutterruptur.

\section{Umstände des Einzelfalles}

Eine Verallgemeinerung des Fahrlässigkeitsbegriffes ist auch deshalb mit Schwierigkeiten verbunden, da stets die «nach den Umständen» gebotene Vorsicht anzuwenden ist [7]. Mangels gesetzlich kodifizierter Standesregeln hat das Bundesgericht festgehalten, dass sich die Sorgfaltspflichten eines Arztes nach der Art des Eingriffs oder Behandlung, den damit verbundenen Risiken sowie den Mitteln und der Dringlichkeit der medizinischen Massnahme, d.h. nach der konkreten Gefahrenlage, richten. Der Arzt hat indes nicht für jene Gefahren und Risiken einzustehen, die immanent mit jeder ärztlichen Handlung und auch mit der Krankheit an sich verbunden sind. Ein Arzt handelt unsorgfältig, wenn sich sein Vorgehen nicht nach den durch die medizinische Wissenschaft aufgestellten und generell anerkannten Regeln richtet und dem jeweiligen Stand der Wissenschaft nicht entspricht. Insofern ist empfehlenswert, dass Ärzte die wissenschaftlichen Fortschritte und Erkenntnisse laufend mitverfolgen, insbesondere im Fachgebiet, in dem ein Spezialist tätig ist.

So wurde ein Arzt der fahrlässigen Körperverletzung schuldig gesprochen, nachdem ein alkoholisierter Mann nach einem harten Sturz auf den Kopf, eingeliefert wurde und sich der behandelnde Arzt auf die Schilderung eines Augenzeugen, der den
Sturz beschönigend darstellte, verliess. Tatsächlich erlitt der Patient einen lebensgefährlichen Schädelbruch, der bleibende Schäden hinterliess. Die Sorgfaltspflichtverletzung des Arztes bestand darin, dass er die Schilderung nicht hinterfragte und die Symptome des Patienten dem übermässigen Alkoholkonsum zuschrieb. Er hätte einen Kopfaufprall stärker in Betracht ziehen und den Patienten engmaschig überwachen müssen, um eine mögliche Blutung im Schädelraum frühzeitig erfassen zu können [8].

\section{Gutachten kommt meist eine präjudizielle Wirkung zu.}

Unserer Auffassung nach ist auch die unterschiedliche Ausstattung an medizinischen Gerätschaften an verschiedenen Spitälern zu berücksichtigen. Demzufolge könnte die konkrete Situation in einem Regionalspital (nämlich zugunsten des beschuldigten Arztes) anders beurteilt werden als dieselbe medizinische Situation (z. B. Komplikation) in einem Zentrumsspital. Auf der anderen Seite haben Ärzte eines Regionalspitals im Wissen um die geringeren Möglichkeiten zeitgerecht die Verlegung eines Patienten in ein Zentrumsspital anzuordnen, um ihrer Sorgfaltspflicht zu genügen.

\section{Übernahmeverschulden}

Nach dem Gesagten wird klar, dass ein Arzt dann nicht zur Verantwortung gezogen werden kann, wenn er überfordert war und seine Überforderung vor dem Hintergrund der Voraussehbarkeit und Vermeidbarkeit sowie anhand seiner persönlichen und fachspezifischen Fähigkeiten gerechtfertigt war. Hat er sich hingegen auf eine Betätigung eingelassen, die bekanntermassen eine besondere Ausbildung oder entsprechende Kenntnisse und Erfahrungen erfordert, oder hat er in anderer Weise eine Aufgabe übernommen, der er nicht gewachsen ist, so kann ihn der Vorwurf des Übernahmeverschuldens treffen. Danach handelt der Arzt auch dann fahrlässig, wenn er die konkreten Gefahren nicht im Einzelnen hat abschätzen können, er aber immerhin in der Lage gewesen wäre zu erkennen, dass ihm die für den entsprechenden Eingriff notwendige Qualifikation oder Erfahrung abgeht [9]. Verfügt demnach ein Arzt nicht über die für die Anamnese oder den Heileingriff erforderlichen Kenntnisse, gehört es zu seiner Sorgfaltspflicht, die weitere Behandlung einem Fachspezialisten zu überlassen.

\section{Bedeutung von Gutachten}

Der Richter stösst im Rahmen der Beurteilung medizinischer Sachverhalte mit seinem Wissen an Gren- 
zen, weshalb die Rechtspraxis regelmässig die Hilfe von Experten in Anspruch nehmen muss, um die Frage der Sorgfaltspflichtverletzung durch einen Arzt beantworten zu können. Für das Urteil gegen einen beschuldigten Arzt sind die Aussagen und Schlussfolgerungen eines medizinischen Gutachters «matchentscheidend». Zur Präzisierung des Fahrlässigkeitsbegriffes bleibt dem Gericht häufig keine andere Wahl, als den Ausführungen des Gutachters zu folgen und die strafrechtliche Sanktion an den wissenschaftlichen Befund zu binden. Da den Gutachten meist eine präjudizielle Wirkung zukommt, muss bereits der Ernennung bzw. der Auswahl der Experten grosse Bedeutung beigemessen werden. Der Strafverteidiger tut gut daran, frühzeitig darauf einzuwirken. Das nahezu «blinde» Folgen der Gerichte bringt es mit sich, dass unseres Erachtens eher ein zu strenger Sorgfaltsmassstab angewendet wird, im Sinne von: Im Nachhinein ist man immer klüger als vorher, vor allem am Richtertisch [10]. delikt zu entkommen. Allerdings sind sie gut beraten, etwa die Spital-Organisation an die aktuellen Bedürfnisse anzupassen, um Mängel im Prozessablauf oder in der zwischenärztlichen und disziplinenübergreifenden Kommunikation zu vermeiden, auch vor dem Hintergrund des Übernahmeverschuldens. Hinsichtlich der Diagnostik ist zu empfehlen, dass die Anamnese in jedem Krankheitsstadium anhand der Symptomatik überdacht werden soll, insbesondere wenn eine Behandlungsmethode nicht (wie gewohnt) anschlägt. Der Vorwurf der Sorgfaltspflichtverletzung kann bei unterlassener Differentialdiagnose und bei Beharren auf der Erstdiagnose für den behandelnden Arzt in strafrechtlicher Hinsicht verheerend sein.

Des Weiteren ist es für einen Arzt unerlässlich, die Patienten im Vorfeld umfassend über die Risiken einer Behandlung aufzuklären. Denn weiss der Patient, worauf er sich einlässt, und willigt er in die Behandlung ein, kann dem Arzt bei entsprechender Ausfüh-

\section{Der Vorwurf der Sorgfaltspflichtverletzung kann bei unterlassener Differentialdiagnose und Beharren auf der Erstdiagnose verheerend sein.}

Die eigentliche Krux an medizinischen Expertisen ist, dass sich die Gutachter oft nicht in die damalige Lage des behandelnden Arztes und in die konkreten Umstände versetzen können. Es fällt auf, dass Gutachten oftmals Ansätze einer Ex-post-Betrachtung aufweisen, anstatt sich juristisch korrekt die medizinische Situation zugrunde zu legen, wie sie der angeschuldigte Arzt vorgefunden hatte. Wahrlich kein leichtes Unterfangen, mit Sicherheit jedoch Aufgabe des Strafverteidigers, auf die staatsanwaltlichen Fragen an den Experten Einfluss zu nehmen.

\section{Mitwirkung anderer Ärzte}

Gerade weil die im Fokus stehenden Tatbestände Fahrlässigkeitsdelikte sind, hat das Zusammenwirken mehrerer Ärzte zur Folge, dass jeder als Täter gilt, wenn er durch sein eigenes unsorgfältiges Handeln zum eingetretenen Erfolg beigetragen hat. Andererseits gilt er als Täter, gleichwohl ob er diese Handlung persönlich vorgenommen oder nur angeordnet hat [11]. Gerade bei Behandlung unter Zuhilfenahme von Fachärzten anderer Disziplinen ist zu untersuchen, ob und gegebenenfalls welchem Arzt bzw. welcher Fachdisziplin die verletzende Handlung zuzuschreiben ist.

\section{Vorsichtsmassnahmen zur Abwendung von Fahrlässigkeitsdelikten}

Angesichts der Mannigfaltigkeit möglicher Konfliktsituationen ist es praktisch unmöglich, den Ärzten eine allgemeingültige Anleitung zu geben, um der Gefahr einer Bezichtigung zu einem Fahrlässigkeits- rung kein Vorwurf gemacht werden. Zudem ist es eminent wichtig, dass Ärzte die Krankengeschichte eines jeden Patienten so ausführlich als nur möglich dokumentieren. Nur so kann sich der Arzt über den Gesundheitszustand des Patienten im Klaren sein, allfällige Prädispositionen richtig einschätzen und verhindern, dass er aus pflichtwidriger Unvorsichtigkeit ein potentielles Risiko nicht bedenkt, was ihm später zum Nachteil gereicht.

Eine gründliche Anamnese, eine situationsbedingt gegebenenfalls gebotene ständige Überwachung nach einer Operation sowie die zeitnahe Einleitung von medizinischen Massnahmen unter Zuhilfenahme von weiteren Fachspezialisten dürften die probatesten Mittel sein, um den Mühlen der Strafjustiz und dem öffentlichen Pranger zu entgehen.

\section{Referenzen}

1 Art. 12 Abs. 3 StGB.

2 Art. 47 Abs. 1 StGB.

3 BGE 110 IV 44.

4 BSK, N 80 zu Art. 12.

5 BSK, N 84 zu Art. 12.

6 BGer 6S.412/2005.

7 Art. 12 Abs. 3 StGB.

8 BGE 130 IV 7ff.

9 BSK, N 82 zu Art. 12.

10 Trechsel/Noll, Schweizerisches Strafrecht Allgemeiner Teil I., § 35 D. 3., Zürich 2004.

11 BSK, N 106 zu Art. 12. 